\section{$\S 24$. Effects of Externally Produced Static Magnetic Island on Edge MHD Modes in the Large Helical Device}

Watanabe, F. (Dep. Energy Eng. Sci., Nagoya Univ.), Toi, K., Ohdachi, S., Sakakibara, S., Morita, S., Narihara, K., Narushima, Y., Morisaki, T., Suzuki, C., Tanaka, K., Tokuzawa, T., Watanabe, K.Y.

In the Large Helical Device (LHD) plasmas, edge MHD instabilities with low mode numbers such as $m / n=1 / 1,3 / 4,2 / 3,3 / 5$ and $1 / 2(m, n$ : poloidal and toroidal mode numbers), which are thought to be ideal/resistive interchange modes, are strongly excited in the edge region of high beta plasmas and plasmas with edge transport barrier (ETB) [1-5]. In the LHD magnetic configuration, a sizable static magnetic island can be externally generated at the $t / 2 \pi=1$ rational surface near the plasma edge, using the perturbation field coils called local island divertor (LID) coil. Edge pressure gradient is modified in the toroidal and poloidal directions by the formation of X-point and O-point of the static island and the achieved volume-averaged beta was appreciably reduced. This is very useful to study effects of static magnetic island on above-mentioned edge MHD modes, because the static island is located near the edge region where a lot of rational surfaces of edge MHD modes are densely packed.

Figure 1 shows an example of $\mathrm{L}-\mathrm{H}$ transition plasma for the case that the large LID field was applied to expand the $m / n=1 / 1$ static magnetic island width. The LID coil current of $I_{\mathrm{LID}} / B_{\mathrm{t}}=+1111 \mathrm{~A} / \mathrm{T}$ was stationary in time throughout the discharge. Phase (I) and (II) show L-mode phase and H-mode phase, respectively. In this shot, the $m / n$ $=1 / 1$ static island grows substantially at the beginning of the phase (III) and destroy the core plasma confinement considerably. Magnetic fluctuations in the phase (III) are dominated by the $m / n=3 / 4$ mode, while the dominant modes activity are $m / n=2 / 3,3 / 4$ and $3 / 5$ one in phase (II).

Dependences of the peak SX-fluctuation amplitude of edge MHD modes at the outboard and inboard edge region $\left(\delta I_{\mathrm{sx}} / I_{\mathrm{sx}}\right)$ on $\left\langle\beta_{\mathrm{dia}}>\right.$ are shown in Fig. 2 for ETB plasmas with and without large LID field. In the case with large LID field, the O- and X-points of the $m / n=1 / 1$ static island can be changed respectively in the outboard or inboard sides, depending on the polarity of the LID coil current. In the case without the LID field, the edge SX fluctuation amplitude in the outboard side is almost same as that in the inboard side. This is consistent with the character of ideal/resistive interchange modes. In the case with the large LID field, the $\delta I_{\mathrm{Sx}} / I_{\mathrm{Sx}}$ in the outboard is much larger than that in the inboard side. It should be noted that this character is also seen in the case the O-point is located in the inboard side with the negative polarity of the LID coil current. That is, the radial structure of edge MHD modes becomes strongly asymmetric along the major radius of a plasma. The radial structure of these edge MHD mode structure seems to have a ballooning like character instead of interchange like one.

\section{Reference}

[1] K. Toi et al., Nucl. Fusion 44, 217 (2004).

[2] K. Toi et al., Phys. P lasmas 12, 020701-1 (2005).
[3] F. Watanabe et al., J. Plasma Fusion Res. 81, 967 (2005).

[4] F. Watanabe et al., Plasma Phys. Control. Fusion 48, A201 (2006).

[5] K. Toi et al., Plasma Phys. Control. Fusion 48, A295 (2006).

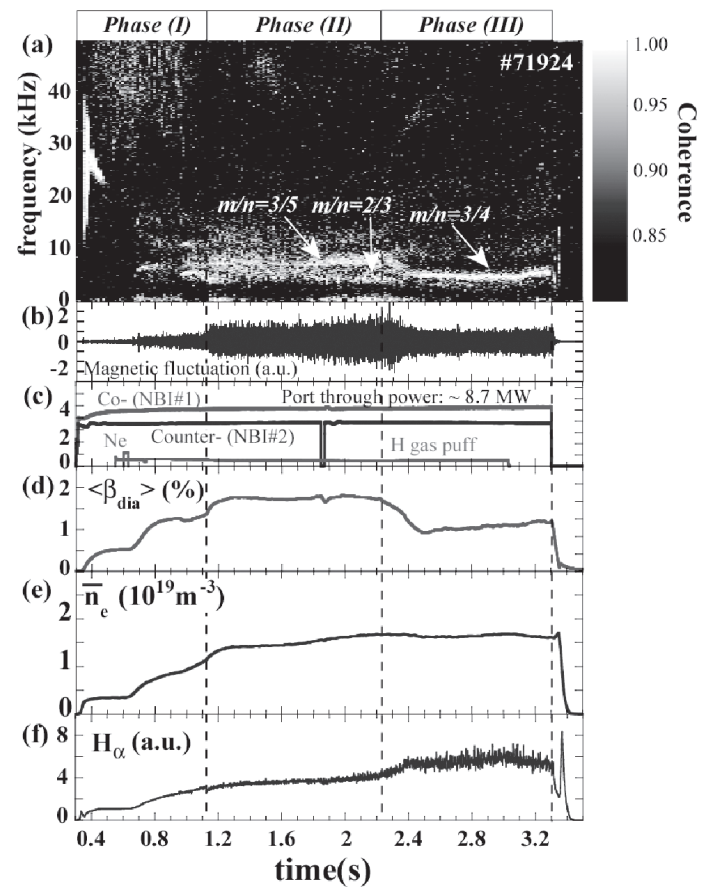

Fig. 1 Time evolution of an L-H transition plasma with the LID field $\left(I_{\mathrm{LID}} / B_{\mathrm{t}}=+1111 \mathrm{~A} / \mathrm{T}\right)$. Phase (I), (II) and (III) show L-mode phase, H-mode phase and core plasma collapse-phase, respectively.

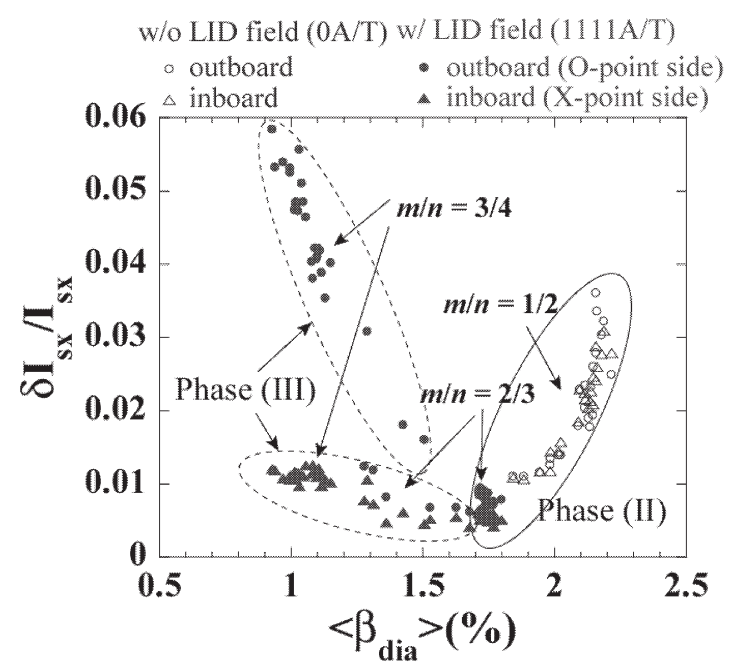

Fig. 2 Dependences of the peak SX fluctuation amplitude related the edge MHD modes in the outboard and inboard sides on the volume averaged beta. $\bigcirc$ : edge SX fluctuations in the outboard side (with O-point side of the $m / n=1 / 1$ static island). $\times$ : edge SX fluctuations in the inboard side (with X-point side of the $m / n=1 / 1$ static island). The area enclosed with solid and dotted lines indicate phase (II) and (III), respectively. 